\title{
PREVALENCE OF VIRULENCE GENES AMONG BULGARIAN NOSOCOMIAL AND CYSTIC FIBROSIS ISOLATES OF PSEUDOMONAS AERUGINOSA
}

\author{
Ivan Mitov, Tanya Strateva*, Boyka Markova
}

Department of Medical Microbiology, Medical University of Sofia, Bulgaria

Submitted: June 20, 2009; Approved: February 18, 2010.

\begin{abstract}
The aim of this study was to evaluate the prevalence of some virulence genes among 202 Pseudomonas aeruginosa isolates from cystic fibrosis (CF) patients $(n=42)$ and non-CF in-patients $(n=160)$ and to analyze the values according to the patient groups, infection localization and antimicrobial resistance. The following frequencies in all studied strains were established: algD (encoding GDP-mannose 6dehydrogenase $\mathrm{AlgD}$ ) - 91.1\%, pilB (type IV fimbrial biogenesis protein PilB) - 23.8\%, nan1 (neuraminidase) $-21.3 \%$, lasB (elastase LasB) - 100\%, plcH (haemolytic phospholipase C precursor) $91.6 \%$, exoS (exoenzyme S) - 62.4\%, and exoU (exoenzyme U) - 30.2\%. The prevalence of nanl was significantly higher $(P<0.01)$ in $\mathrm{CF}$ isolates $(38.1 \%)$ than that in non-CF isolates $(16.9 \%)$. The nanlpositive CF strains were cultured from 16 patients with recurrent lung exacerbations. This study revealed a statistically significant difference $(P<0.01)$ between the portion of multidrug-resistant $(\mathrm{MDR})$ nosocomial $P$. aeruginosa strains containing a large number $(\geq 5)$ of virulence genes $(38.1 \%)$ and the respective part of non-MDR isolates $(17.6 \%)$. Moreover, pilB, exoU and nanl manifested a higher spread $(P<0.001)$ among MDR than in non-MDR strains (respectively, $39.1 \%$ vs. $13.2 \%$; $40.2 \%$ vs. $17.7 \%$ and $26.1 \%$ vs. $4.4 \%$ ). In conclusion, the dissemination of nanl in $\mathrm{CF}$ isolates was moderate and correlated with the lower proportion of patients with lung exacerbations. The molecular-genetic detection of this gene may be used as an indirect measure of CF pulmonary disease evolution. Simultaneous determination of virulence factors and antimicrobial resistance is the contemporary approach for examination of the microbiological aspects of infections caused by $P$. aeruginosa.
\end{abstract}

Key words: Pseudomonas aeruginosa, virulence genes, polymerase chain reaction, cystic fibrosis isolates, non-cystic fibrosis isolates.

\section{INTRODUCTION}

Pseudomonas aeruginosa is an opportunistic pathogen that is a common cause of hospital-acquired infections, particularly infecting patients with predisposing factors, such as burn victim, immunocompromised hosts, or those with metabolic disorders. In cystic fibrosis (CF) patients, P. aeruginosa is believed to be a major contributory factor to chronic lung infections, which could form biofilm and adhere to human mucin in the lower respiratory tract (23). The organism can 
only be eradicated in the early stage of colonization, while reduction of bacterial density is desirable during chronic colonization or exacerbations (3). It is not surprising that $P$. aeruginosa infections are associated with significant morbidity and mortality due to the organism's capacity to adapt easily to changes in the environment, to rapidly develop resistance to antibiotics, and to produce a variety of virulence factors.

$P$. aeruginosa possesses an "arsenal" of cell-associated and extracellular virulence factors. Many extracellular virulence factors have been shown to be controlled by a complex regulatory circuit involving cell-to-cell signaling (quorum sensing) systems that allow the bacteria to produce these factors in a coordinated, cell-density-dependent manner (22).

The aim of this study was to examine the prevalence of virulence genes (encoding adhesins, neuraminidase, invasins and type III effector proteins) in clinical isolates of $P$. aeruginosa and to analyze the values in respect to the patient groups, infection localization and antimicrobial resistance of the strains.

\section{MATERIALS AND METHODS}

\section{Bacterial isolates and patients}

A total of 202 clinical isolates of $P$. aeruginosa (including 160 nosocomial and $42 \mathrm{CF}$ ) was used in the present study. The nosocomial isolates were cultured during 2001-2007 from hospitalized non-CF patients $(n=160)$ of different types of ward in six large hospitals in Sofia, Bulgaria. They were obtained from urine $(n=62)$, tracheal aspirates $(n=22)$, bronchial lavage $(n=12)$, sputum $(n=9)$, pleural fluid $(n=2)$, surgical wounds or abscesses $(n=26)$, blood $(n=8)$, nose $(n=8)$, throat $(n=8)$, and bile $(\mathrm{n}=3)$. The CF P. aeruginosa isolates were recovered from sputa of 42 patients ( 25 females and 17 males) aged 6-27 years during the period 2005-2008. Bacterial identification was performed using a BBL Enteric/Nonfermenter ID system (Becton Dickinson).

\section{CF exacerbation diagnostic criteria}

An acute exacerbation was defined, as per the 1994 Cystic
Fibrosis Foundation (CFF) Microbiology Consensus Conference (4), as the presence of at least 3 of 11 new clinical findings, including increased cough, increased sputum production, fever, weight loss, school or work absenteeism, increased work of breathing, decreased exercise tolerance, or a deterioration in the chest exam, chest radiograph, forced expiratory volume in $1 \mathrm{~s}\left(\mathrm{FEV}_{1}\right)$, or hemoglobin saturation.

\section{Definition of multidrug-resistant (MDR) isolates}

Multidrug-resistant (MDR) P. aeruginosa isolates were defined as resistant to at least three of the following antibiotics: ceftazidime, imipenem, gentamicin and ciprofloxacin (14), using the conventional serial agar dilution method. The minimal inhibitory concentrations were interpreted according to the Clinical and Laboratory Standards Institute (CLSI) 2007 guide-line (5).

\section{Polymerase chain reaction (PCR) amplification of virulence genes}

The prevalence of virulence genes, encoding GDP mannose 6-dehydrogenase (alginate) ( $\operatorname{llg} D)$, type IV fimbrial biogenesis protein PilB (pilB), neuraminidase (nanl), elastase LasB (lasB), haemolytic phospholipase $\mathrm{C}$ precursor (plcH), exoenzyme $\mathrm{S}(\operatorname{exoS})$ and exoenzyme $\mathrm{U}($ exoU), was determined by PCR. The virulence genes were amplified with specific primers (Alpha DNA) listed in Table 1. DNA from the isolates was extracted by boiling. PCR was carried out with $2 \mu \mathrm{l}$ template DNA, $0.25 \mu \mathrm{M}$ of each primer, $0.2 \mu \mathrm{mM}$ deoxyribonucleoside triphosphates, $1 \mathrm{x}$ reaction buffer, $2 \mathrm{mM}$ $\mathrm{MgCl}_{2}$ and $1.5 \mathrm{U}$ Prime Taq DNA polymerase (GeNet Bio) in a total volume of $25 \mu \mathrm{l}$. The DNA was amplified using the following protocol: initial denaturation $\left(94{ }^{\circ} \mathrm{C}\right.$ for $\left.5 \mathrm{~min}\right)$, followed by $25-30$ cycles of denaturation $\left(94^{\circ} \mathrm{C}\right.$ for $\left.35-45 \mathrm{~s}\right)$, annealing $\left(53-62^{\circ} \mathrm{C}\right.$, from $45 \mathrm{~s}$ to $\left.1 \mathrm{~min}\right)$ and extension $\left(72{ }^{\circ} \mathrm{C}\right.$, from $45 \mathrm{~s}$ to $1 \mathrm{~min} 35 \mathrm{~s}$ ), with a single final extension of $7 \mathrm{~min}$ at $72{ }^{\circ} \mathrm{C}$. PCR products were separated in $1 \%$ agarose gel for 50-110 min at $130 \mathrm{~V}$, stained with ethidium bromide $(0.5 \mu \mathrm{g} / \mathrm{ml})$ and detected by UV transillumination (wavelength $312 \mathrm{~nm})$. Amplified genes were identified on the basis of fragment size shown in Table 1. 
Table 1. Primers used for amplification of virulence genes.

\begin{tabular}{|c|c|c|c|c|c|c|}
\hline Primers & $\begin{array}{c}\text { Target } \\
\text { gene }\end{array}$ & Sequence $\left(5^{\prime}-3^{\prime}\right)$ & $\begin{array}{l}\text { Product } \\
\text { size (bp) }\end{array}$ & $\begin{array}{c}\text { Position on } \\
P . \text { aeruginosa PAO1 } \\
\text { chromosome (locus) }\end{array}$ & $\begin{array}{c}\text { Annealing } \\
\text { temperature } \\
\left({ }^{\circ} \mathrm{C}\right)\end{array}$ & Source \\
\hline algD-F & \multirow[t]{2}{*}{$\operatorname{alg} D$} & ATG CGA ATC AGC ATC TTT GGT & \multirow[t]{2}{*}{1310} & \multirow[t]{2}{*}{ PA3540 } & \multirow[t]{2}{*}{62} & \multirow[t]{2}{*}{$(15)$} \\
\hline algD-R & & CTA CCA GCA GAT GCC CTC GGC & & & & \\
\hline pilB-F & \multirow[t]{2}{*}{ pilB } & ATG AAC GAC AGC ATC CAA CT & \multirow[t]{2}{*}{826} & \multirow[t]{2}{*}{ PA4526 } & \multirow[t]{2}{*}{60} & \multirow[t]{2}{*}{$(20)$} \\
\hline pilB-R & & GGG TGT TGA CGC GAA AGT CGA T & & & & \\
\hline nan1-R & nanl & CTA AAT CCA TGC TCT GAC CC & 1317 & PA2794 & 53 & $(20)$ \\
\hline lasB-F & \multirow[t]{2}{*}{ las $B$} & GGA ATG AAC GAG GCG TTC TC & \multirow[t]{2}{*}{300} & \multirow[t]{2}{*}{ PA3724 } & \multirow[t]{2}{*}{60} & \multirow[t]{2}{*}{$(15)$} \\
\hline lasB-R & & GGT CCA GTA GTA GCG GTT GG & & & & \\
\hline plcH-F & plcH & GAA GCC ATG GGC TAC TTC AA & 307 & PA0844 & 60 & $(15)$ \\
\hline exoU-F & \multirow[t]{2}{*}{ exoU } & GGG AAT ACT TTC CGG GAA GTT & \multirow[t]{2}{*}{428} & PS14 within & \multirow[t]{2}{*}{60} & \multirow[t]{2}{*}{$(20)$} \\
\hline exoU-R & & CGA TCT CGC TGC TAA TGT GTT & & $\begin{array}{c}\text { P. aeruginosa } \text { PA14 } \\
\text { pathogenicity island } \\
\text { PAPI-2 }\end{array}$ & & \\
\hline
\end{tabular}

PCR, polymerase chain reaction; $\mathrm{F}$, forward; $\mathrm{R}$, reverse.

algD, GDP-mannose 6-dehydrogenase AlgD (alginate)-encoding gene; pilB, type IV fimbrial biogenesis protein PilB-encoding gene; nan1, neuraminidase-encoding gene; $p l c H$, haemolytic phospholipase $\mathrm{C}$ precursor-encoding gene; las $B$, elastase LasB-encoding gene; exoS, exoenzyme $\mathrm{S}$-encoding gene; exo $U$, exoenzyme $\mathrm{U}$ encoding gene.

\section{Statistical analysis}

The distribution of virulence genes with respect to isolate origin was compared using the chi-square test. A $P$ value below 0.05 was considered to be statistically significant.

\section{RESULTS}

\section{Prevalence of virulence genes among all studied $P$. aeruginosa isolates}

The frequencies of occurrence of virulence genes in all studied strains $(\mathrm{n}=202)$ were as follows: $\operatorname{alg} D-91.1 \%$, pilB $23.8 \%$, nanl $-21.3 \%$, lasB $-100 \%$, plcH $-91.6 \%$, exoS $62.4 \%$, and $e x o U-30.2 \%$.

Prevalence of virulence genes among $C F$ and non-CF $P$. aeruginosa isolates

The comparative frequencies of virulence genes in nosocomial non-CF isolates $(\mathrm{n}=160)$ and $\mathrm{CF} P$. aeruginosa isolates ( $\mathrm{n}=42)$ were as follows: $\operatorname{alg} D-92.5 \%$ vs. $85.7 \%$, pilB $-26.9 \%$ vs. $9.5 \%$, nanl $-16.9 \%$ vs. $38.1 \%$, lasB $-100 \%$ for both type of isolates, $\mathrm{plcH}-96.9 \%$ / 71.4\%, exoS $-65.0 \%$ / $52.4 \%$, and $e x o U-30.6 \% / 28.6 \%$.

(i) A statistically significant difference was not found between the dissemination of $\operatorname{alg} D, \operatorname{las} B, \operatorname{exoS}$ and $\operatorname{exo} U$ among the two groups of patients monitored.

(ii) The prevalence of nanl was significantly higher in $\mathrm{CF}$ isolates $(38.1 \%)$ than that in non-CF isolates of $P$. aeruginosa $(16.9 \%)-P<0.01$. The nanl-positive $\mathrm{CF}$ isolates $(n=16)$ were obtained from 16 patients with recurrent pulmonary exacerbations. Exacerbations were manifested by: weight loss of at least $1 \mathrm{~kg}$; acute changes in respiratory signs and symptoms, such as increased cough frequency, sputum production and dyspnea, new auscultation findings (crackles, wheezing, rales or ronchi); inflammation changes according to the complete blood count (elevated peripheral leukocyte 
count (more than $9.5 \times 10^{9}$ white blood cells/L) and erythrocyte sedimentation rate over $17 \mathrm{~mm} / \mathrm{h}$ ) and increased serum C-reactive protein levels (higher than $3.9 \mathrm{mg} / \mathrm{L}$ ); new changes on X-ray, such as pneumonia and emphysema (found in $81 \%$ of the patients); and elevated levels of polynucler cells and metaplasia on sputum cytology (in $75 \%$ of them). In addition, the drop of forced vital capacity (FVC) and $\mathrm{FEV}_{1}$ was drastic and hardly came back at baseline after the treatment. Unfortunately, in 2008 two children died after severe lung exacerbations.

(iii) The detection of pilB and $\mathrm{plcH}$ among $\mathrm{CF}$ isolates was significantly lower than that in non-CF nosocomial strains $(P<0.01$ and $P<0.001$, respectively).

\section{Prevalence of virulence genes with respect to the infection}

\section{localization in non-CF patients}

The distribution of virulence genes encoding adhesins (alginate, type IV pili), neuraminidase, invasins (elastase LasB, haemolytic phospholipase C) and type III effector proteins (ExoS, ExoU) varied in respect to the infection localization in non-CF patients (Table 2).

(i) The highest spread of pilB was established among $P$. aeruginosa isolates from blood (75.0\%) and urines $(35.5 \%)$, which was significantly higher than that in isolates from in-patients with upper respiratory tract infections (URTIs) (12.5\%) and lower respiratory tract infections (LRTIs) $(11.1 \%)-P<0.001$ and $P<0.05$,
$P<0.01$, respectively.

(ii) The nanl gene revealed a widespread dissemination in $P$. aeruginosa isolates from blood (62.5\%), which was significantly higher than that among the isolates from urines $(19.4 \%)-P<0.02$; wounds $(15.4 \%)-P<0.02$; URTIs $(12.5 \%)-P<0.01$; and LRTIs $(6.7 \%)-P<0.01$.

(iii) The presence of exoS was the highest among $P$. aeruginosa isolates from blood (87.5\%) and significantly different only with those obtained from in-patients with LRTIs $(55.6 \%)-P<0.05$.

(iv) The exoU frequencies were the most expressive in the wound $(46.2 \%)$ and LRTIs $P$. aeruginosa isolates $(40.0 \%)$. They were significantly higher than those of the isolates from urines $(17.7 \%)-P<0.01$ and $P<0.02$, respectively; and blood $(12.5 \%)-P<0.05$.

\section{Prevalence of virulence genes among MDR and non-MDR}

\section{P. aeruginosa isolates from non-CF patients}

Of the 160 nosocomial non-CF P. aeruginosa isolates, 92 were MDR. Three of the genes (pilB, nanl and exoU) manifested a significantly higher spread $(P<0.001)$ among MDR than in non-MDR strains of $P$. aeruginosa (respectively, $39.1 \%$ vs. $13.2 \% ; 26.1 \%$ vs. $4.4 \%$ and $40.2 \%$ vs. $17.7 \%$ ) Figure 1.

The proportion of MDR nosocomial P. aeruginosa isolates containing a large number of virulence genes $(\geq 5)(38.1 \%)$ was higher than the proportion of non-MDR isolates with a large number of virulence genes $(17.6 \%)-P<0.01$ (Table 3).

Table 2. Prevalence (as percentage) of virulence genes in non-cystic fibrosis $P$. aeruginosa isolates $(\mathrm{n}=160)$.

\begin{tabular}{|c|c|c|c|c|c|c|}
\hline \multirow[t]{2}{*}{ Gene } & \multicolumn{6}{|c|}{ Non-cystic fibrosis isolates } \\
\hline & $\begin{array}{c}\text { Urine } \\
(\mathrm{n}=62)\end{array}$ & $\begin{array}{l}\text { LRTIs } \\
(n=45)\end{array}$ & $\begin{array}{l}\text { URTIs } \\
(n=16)\end{array}$ & $\begin{array}{c}\text { Wounds } \\
(n=26)\end{array}$ & $\begin{array}{l}\text { Blood } \\
(\mathbf{n}=8)\end{array}$ & $\begin{array}{c}\text { Total }^{a} \\
(\mathrm{n}=160)\end{array}$ \\
\hline $\operatorname{alg} D$ & 93.5 & 95.6 & 93.8 & 84.6 & 100.0 & 92.5 \\
\hline pilB & 35.5 & 11.1 & 12.5 & 26.9 & 75.0 & 26.9 \\
\hline nanl & 19.4 & 6.7 & 12.5 & 15.4 & 62.5 & 16.9 \\
\hline las $B$ & 100.0 & 100.0 & 100.0 & 100.0 & 100.0 & 100.0 \\
\hline plcH & 96.8 & 97.8 & 100.0 & 96.2 & 100.0 & 96.9 \\
\hline $\operatorname{exos}$ & 66.1 & 55.6 & 75.0 & 65.4 & 87.5 & 65.0 \\
\hline exoU & 17.7 & 40.0 & 37.5 & 46.2 & 12.5 & 30.6 \\
\hline
\end{tabular}

${ }^{a}$ Frequency of the virulence gene in all non-cystic fibrosis isolates of $P$. aeruginosa, including three bile isolates.

LRTIs, lower respiratory tract infections; URTIs, upper respiratory tract infections; algD, GDP-mannose 6-dehydrogenase AlgD (alginate)-encoding gene; pilB, type IV fimbrial biogenesis protein PilB-encoding gene; nan 1, neuraminidase-encoding gene; plcH, haemolytic phospholipase C precursorencoding gene; las $B$, elastase LasB-encoding gene; exo $S$, exoenzyme S-encoding gene; exoU, exoenzyme U-encoding gene. 


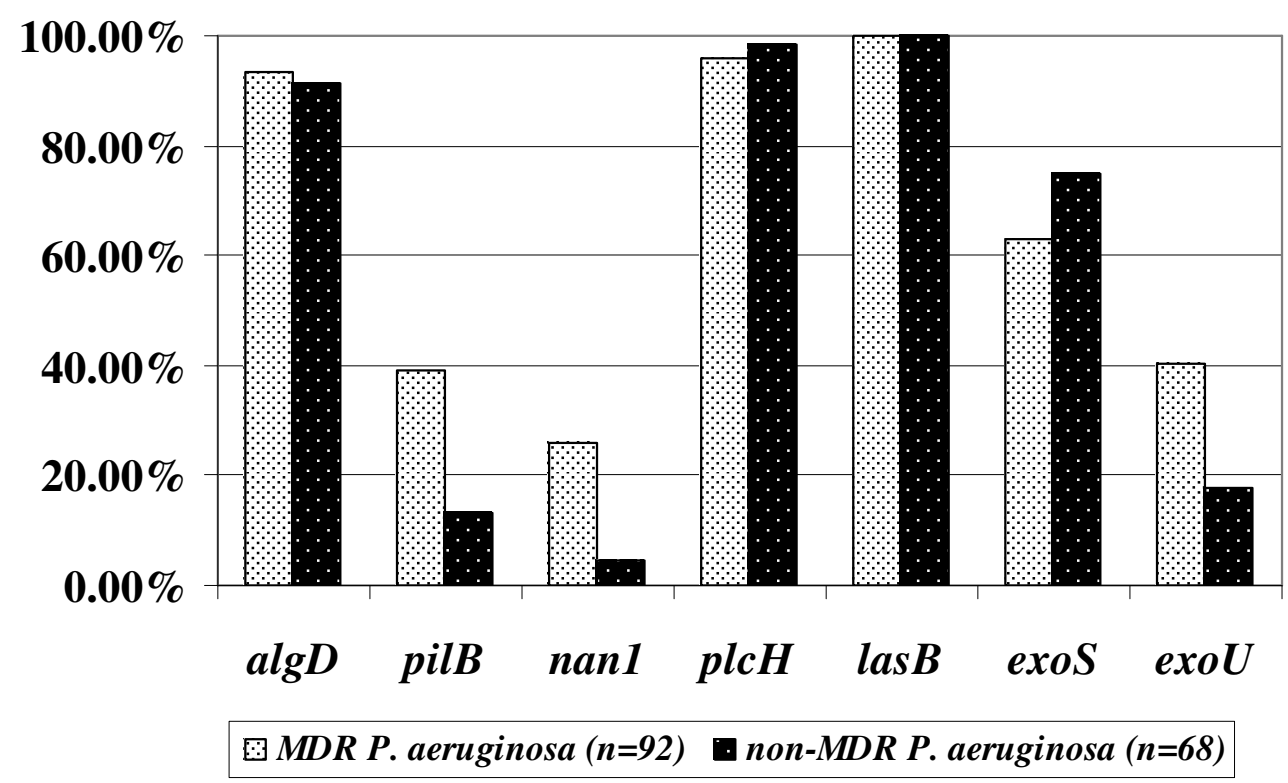

Figure 1. Prevalence (as percentages) of virulence genes among MDR and non-MDR P. aeruginosa isolates from non-cystic fibrosis patients. MDR, multidrug-resistant; algD, GDP-mannose 6-dehydrogenase AlgD (alginate)-encoding gene; pilB, type IV fimbrial biogenesis protein PilB-encoding gene; nan1, neuraminidase-encoding gene; plcH, haemolytic phospholipase $\mathrm{C}$ precursor-encoding gene; las $B$, elastase LasB-encoding gene; exoS, exoenzyme S-encoding gene; exoU, exoenzyme U-encoding gene.

Table 3. Presence of virulence genes among MDR and non-MDR nosocomial P. aeruginosa isolates.

\begin{tabular}{lcc}
\hline $\begin{array}{l}\text { Number of } \\
\text { virulence genes }\end{array}$ & $\begin{array}{c}\text { Number }(\%) \text { of } \\
\text { MDR isolates }(\mathbf{n = 9 2 )}\end{array}$ & $\begin{array}{c}\text { Number (\%) of } \\
\text { non-MDR isolates (n=68) }\end{array}$ \\
\hline 1 & $1(1.1)$ & $1(1.5)$ \\
2 & $1(1.1)$ & $0(0.0)$ \\
3 & $13(14.1)$ & $7(10.3)$ \\
4 & $42(45.7)$ & $48(70.6)$ \\
5 & $16(17.4)$ & $10(14.7)$ \\
6 & $17(18.5)$ & $2(2.9)$ \\
7 & $2(2.2)$ & $0(0.0)$ \\
\hline
\end{tabular}

MDR, multidrug-resistant isolate.

\section{DISCUSSION}

The established prevalence of some of the genes $(\operatorname{alg} D$, las $B$ and $p l c H$ ) among all studied $P$. aeruginosa isolates $(n=202)$ was similar to their widespread dissemination $(100 \%)$ in French $P$. aeruginosa isolates $(\mathrm{n}=162)$ obtained from sputa of CF patients, clinical samples from patients without inherited diseases and plants (15). In our study the frequencies of virulence genes encoding type III secretion proteins - ExoS $(62.4 \%)$ and ExoU (30.3\%), were similar to those ascertained by Feltman et al. (8) - respectively, $72 \%$ and $28 \%$ among CF and non-CF P. aeruginosa isolated in USA during 1999-2000. The part of our $P$. aeruginosa strains containing either the exoS (51\%) or the exoU gene $(18.8 \%)$ was higher than that of isolates having both genes (exoS+exoU) $-11.4 \%$. Previously, Feltman et al. (8) reported that of 115 P. aeruginosa clinical 
and environmental isolates, 82 contained the exoS but not the exoU gene, 31 contained the exoU but not the exoS gene and a single contained both genes. However, recent studies established that the simultaneous exoS+exoU detection was a frequent finding in clinical and environmental $P$. aeruginosa isolates. Finnan et al. (9) found $75 \%$ dissemination of both genes.

It was not surprising that the detection of nanl among the investigated CF $P$. aeruginosa was significantly higher than that in non-CF isolates. Three decades ago, neuraminidase production by a CF $P$. aeruginosa isolate was described and suggested to contribute to lung infection (16). The spread of nanl in CF P. aeruginosa isolates increases when the clinical state of patients worsens, suggesting the possible role of neuraminidase in $\mathrm{CF}$ pulmonary disease evolution $(15,21)$. The prevalence of nanl among our CF isolates was significantly lower $(P<0.02)$ than that in French CF $P$. aeruginosa isolates examined by Lanotte et al. (15) - 38.1\% / $61.7 \%$, which was related to their predominant stable clinical status. On auscultation they had a clear vesicular murmur (with bronchial character in the places where bronchiectasis was formed) without any rales or ronchi. All data from complete blood count were within normal limits or on the upper limit. There were no additional new changes on X-ray findings. The spirometry showed steady levels of forced vital capacity and $\mathrm{FEV}_{1}$, slightly decreasing with the age. The sputum cytology manifested steady levels of polynuclear cells and the rate of metaplasia was not increasing significantly with check-ups.

In the present study, exoS gene was found more rarely in $\mathrm{CF}$ isolates than in the other clinical isolates $(52.4 \%$ vs. $65.0 \%$ ), which contrasted with the reported higher secretion of ExoS by $P$. aeruginosa strains from sputa of CF individuals (6, 8, 15). According to the data published by Lanotte et al. (15), the prevalence of exoS among CF P. aeruginosa isolates was significantly higher than that in non-CF isolates $(93.8 \%$ vs. $75.3 \% ; P=0.002$ ). Feltman et al. (8) reported $85 \%$ distribution of exoS among isolates from sputa of $\mathrm{CF}$ patients and a variable frequency of the gene in the clinical strains from samples of patients without inherited diseases (from $60 \%$ to
$75 \%)$.

The frequency of pilB among the studied CF $P$. aeruginosa was low (9.5\%) which suggested the role of nonpilus adhesins (such as alginate) in this group of isolates. Recently, it was ascertained an absolute absence of pilB among CF P. aeruginosa strains from Ireland (9).

As it was described above, the distribution of virulence genes varied in respect to the site of infection among non-CF in-patients. The nanl-detection (from $6.7 \%$ (LRTIs) to $62.5 \%$ (blood)) was lower than that in similar French clinical strains of $P$. aeruginosa (40.7\% - urines, $48 \%$ - LRTIs, $41.2 \%$ wounds) (15).

In this study, the prevalence of exoS among LRTIs isolates (55.6\%) was lower than that in LRTIs P. aeruginosa isolates from the studies mentioned above (Lanotte et al. $-80 \%$, and Feltman et al. - 75\%) (8, 15). ExoS is responsible for direct tissue destruction in lung infection and may be important for bacterial dissemination (22).

exoU was found predominantly in $P$. aeruginosa isolates from LRTIs and wounds. Its prevalence in our wound isolates (46.2\%) was nearly identical with that found by Feltman et al. in USA (40.0\%) (8). It is known that $90 \%$ of ExoU-producing $P$. aeruginosa strains are associated with severe infections (11). Of the type III secretion proteins, ExoU is the most cytotoxic. Secretion of ExoU is a marker for highly virulent $P$. aeruginosa isolates obtained from patients with hospitalacquired pneumonia (18).

Our work revealed a significant difference between the portion of MDR nosocomial $P$. aeruginosa strains containing a large number $(\geq 5)$ of virulence genes and the respective part of non-MDR isolates. Numerous reports have documented that the rise in MDR nosocomial $P$. aeruginosa continues to threaten hospitalized patients despite various countermeasures including isolation techniques and antibiotic de-escalation therapy $(2,13)$. Whether MDR P. aeruginosa strains necessarily express a more virulent phenotype continues to remain a controversial issue (7). Recently, Zaborina et al. screened consecutively isolated MDR P. aeruginosa clinical strains for their ability to disrupt the integrity of human 
cultured intestinal epithelial cells (Caco-2) and correlated these finding to related virulence phenotypes such as adhesiveness, motility, biofilm formation and cytotoxicity (24). These strains were characterized and found to harbor the exoU gene and to display high swimming motility and adhesiveness.

It is known that bacteria growing in biofilms are more resistant to antimicrobial agents than their planktonic counterparts are (12). Various hypotheses have been put forward to explain biofilm resistance (poor antibiotic penetration, nutrient limitation and slow growth, high cell density, adaptive stress responses etc.), but to date, there are no data that entirely explain this phenomenon (19). Due to the success of a genetic screen that Mah et al. carried out, some additional mechanisms are being proposed. The locus identified in their screen, $n d v B$, is required for the synthesis of periplasmic glucans. These glucose polymers may prevent antibiotics from reaching their sites of action by sequestering these antimicrobial agents in the periplasm (17).

We presented a significantly higher spread of some virulence genes (exoU, pilB and nanl) among MDR nosocomial $P$. aeruginosa isolates. In a recent accomplished study, Garey et al. determined the prevalence of exoU and exoS from bloodstream isolates of hospitalized patients with $P$. aeruginosa bacteremia and ascertained that the isolates containing the exoU gene were significantly more resistant $(P<0.05)$ to different classes of antimicrobials: $\beta$-lactams (piperacillin/tazobactam, ceftazidime, cefepime, carbapenems), fluoroquinolones, and aminoglycosides (gentamicin) (10).

In conclusion, the present study established a higher frequency of spread of nanl among CF P. aeruginosa isolates compared with that in non-CF isolates. This prevalence was moderate and correlated with the lower proportion of patients with lung exacerbations. The molecular-genetic detection of the nanl gene may be used as an indirect measure of $\mathrm{CF}$ pulmonary disease evolution. The distribution of virulence genes varied in respect to the site of infections among non- $\mathrm{CF}$ hospitalized patients. For the first time, it was found that the frequencies of pilB, exoU and nanl were significantly higher in MDR strains than those in non-MDR P. aeruginosa strains.
Moreover, the part of MDR isolates containing a large number of virulence factors $(\geq 5)$ was bigger than the respective partition of non-MDR $P$. aeruginosa. The exact reasons for the frequent presence of virulence-related genes in MDR isolates are still unknown therefore a further work is necessary. Simultaneous determination of virulence factors and antimicrobial resistance is a contemporary approach for examination of the microbiological aspects of infections caused by $P$. aeruginosa.

\section{ACKNOWLEDGEMENT}

The study was supported by a grant from the Medical University of Sofia (Council of Medical Science, project no. 19/2007, grant no. 33/2007).

\section{REFERENCES}

1. Allewelt, M.; Coleman, F.T.; Grout, M.; Priebe, G.P.; Pier, G.B. (2000). Acquisition of expression of the Pseudomonas aeruginosa ExoU cytotoxin leads to increased bacterial virulence in a murine model of acute pneumonia and systemic spread. Infect. Immun. 68 (7), 3998-4004.

2. Alonso, A.; Campanario, E.; Martinez, J.L. (1999). Emergence of multidrugresistant mutants is increased under antibiotic selective pressure in Pseudomonas aeruginosa. Microbiology 145 (10), 28572862.

3. Canton, R.; Cobos, N.; de Gracia J.; Baquero, F.; Honorato, J.; Gartner, S.; Alvarez, A.; Salcedo, A.; Oliver, A.; Garcia-Quetglas, E. on behalf of the Spanish Consensus Group for Antimicrobial Therapy in the Cystic Fibrosis Patient. (2005). Antimicrobial therapy for pulmonary pathogenic colonization and infection by Pseudomonas aeruginosa in cystic fibrosis patients. Clin. Microbiol. Infect. 11 (9), 690-703.

4. CFF. (1994). Microbiology and infectious disease in cystic fibrosis. Consensus Conference. Concepts in Care Vol. 5, section 1, Bethesda MD: Cystic Fibrosis Foundation.

5. CLSI. (2007). Performance standards for antimicrobial susceptibility testing, $17^{\text {th }}$ informational supplement, M100-S17. Wayne PA: Clinical and Laboratory Standards Institute.

6. Dacheux, D.; Toussaint, B.; Richard, M.; Brochier, G.; Croize, J.; Attree, I. (2000). Pseudomonas aeruginosa cystic fibrosis isolates induce rapid, type III secretion-dependent, but ExoU-independent, oncosis of macrophages and polymorphonuclear neutrophils. Infect. Immun. 68 (5), 2916-2924. 
7. Di Martino, P.; Gagniere, H.; Berry, H.; Bret, L. (2002). Antibiotic resistance and virulence properties of Pseudomonas aeruginosa strains from mechanically ventilated patients with pneumonia in intensive care units: comparison with imipenem-resistant extra-respiratory tract isolates from uninfected patients. Microbes Infect. 4 (6), 613-620.

8. Feltman, H.; Schulert, G.; Khan, S.; Jain, M.; Peterson, L.; Hauser, A.R. (2001). Prevalence of type III secretion genes in clinical and environmental isolates of Pseudomonas aeruginosa. Microbiology 147 (10), 2659-2669.

9. Finnan, S.; Morrissey, J.P.; O’Gara, F.; Boyd, E.F. (2004). Genome diversity of Pseudomonas aeruginosa isolates from cystic fibrosis patients and the hospital environment. J. Clin. Microbiol. 42 (12), 5783-5792.

10. Garey, K.W.; Vo, Q.P.; Larocco, M.T.; Gentry, L.O.; Tam, V.H. (2008). Prevalence of type III secretion protein exoenzymes and antimicrobial susceptibility patterns from bloodstream isolates of patients with Pseudomonas aeruginosa bacteremia. J. Chemother. 20 (6), 714-720.

11. Hauser, A.R.; Cobb, E.; Bodi, M.; Mariscal, D.; Valles, J.; Engel, J.N.; Rello, J. (2002). Type III protein secretion is associated with poor clinical outcomes in patients with ventilator-associated pneumonia caused by Pseudomonas aeruginosa. Crit. Care Med. 30 (3), 521-528.

12. Hoyle, B.D.; Costeron, W.J. (1991). Bacterial resistance to antibiotics: the role of biofilms. Prog. Drug Res. 37, 91-105.

13. Jones, R.N. (2001). Resistance patterns among nosocomial pathogens: trends over the past few years. Chest 119 (2 Suppl.), 397S-404S.

14. Karlowsky, J.A.; Draghi, D.C.; Jones, M.E.; Thornsberry, C.; Friedland, I.R.; Sahm, D.F. (2003). Surveillance for antimicrobial susceptibility among clinical isolates of Pseudomonas aeruginosa and Acinetobacter baumannii from hospitalized patients in the United States, 1998-2001. Antimicrob. Agents Chemother. 47 (5), 1681-1688.

15. Lanotte, P.; Watt, S.; Mereghetti, L.; Dartiguelongue, N.; RastegarLari, A.; Goudeau, A.; Quentin, R. (2004). Genetic features of Pseudomonas aeruginosa isolates from cystic fibrosis patients compared with those of isolates from other origins. J. Med. Microbiol. 53 (1), 73-81.

16. Leprat, R.; Michel-Briand, Y. (1980). Extracellular neuraminidase production by a strain of Pseudomonas aeruginosa isolated from cystic fibrosis. Ann. Microbiol. 131B (3), 209-222.

17. Mah, T.F.; Pitts, B.; Pellock, B.; Walker, G.C.; Stewart, P.S.; O'Toole G.A. (2003). A genetic basis for Pseudomonas aeruginosa biofilm antibiotic resistance. Nature 426 (6964), 306-310.

18. Schulert, G.S.; Feltman, H.; Rabin, S.D.; Martin, C.G.; Battle, S.E.; Rello, J.; Hauser A.R. (2003). Secretion of the toxin ExoU is a marker for highly virulent Pseudomonas aeruginosa isolates obtained from patients with hospital-acquired pneumonia. J. Infect. Dis. 188 (11), 1695-1706.

19. Stewart, P.C. (2002). Mechanisms of antibiotic resistance in bacterial biofilms. Int. J. Med. Microbiol. 292 (2), 107-113.

20. Strateva, T. (2008). Microbiological and molecular-genetic investigations on resistance mechanisms and virulence factors in clinical strains of Pseudomonas aeruginosa. Sofia, Bulgaria, 210 p. (Ph.D. Dissertation, Medical University of Sofia, Bulgaria).

21. Strateva, T.; Petrova, G.; Perenovska, P.; Mitov, I. (2009). Bulgarian cystic fibrosis Pseudomonas aeruginosa isolates: antimicrobial susceptibility and neuraminidase-encoding gene distribution. J. Med. Microbiol. 58 (5), 690-692.

22. Van Delden, C.; Iglewski, B.H. (1998). Cell-to-cell signaling and Pseudomonas aeruginosa infections. Emerg. Infect. Dis. 4 (4), 551560.

23. Whiteley, M.; Bangera, M.G.; Bumgarner, R.E.; Parsek, M.R.; Teitzel, G.M.; Lory, S.; Greenberg, E.P. (2001). Gene expression in Pseudomonas aeruginosa biofilms. Nature 413 (6858), 860-864.

24. Zaborina, O.; Kohler, J.E.; Wang, Y.; Bethel, C.; Shevchenko, O.; Wu, L.; Turner, J.R.; Alverdy, J.C. (2006). Identification of multi-drug resistant Pseudomonas aeruginosa clinical isolates that are highly disruptive to the intestinal epithelial barrier. Ann. Clin. Microbiol. Antimicrob. 5, 14 